\title{
OSMOTIC SHRINKAGE
}

\section{OF GIANT EGG-LECITHIN VESICLES}

\author{
E. Boroske ANd M. ElwenSPoek, Freie Universität Berlin, Institut für Atom- und \\ Festkörperphysik, Königin-Luise-Str. 28/30, D-1000 Berlin 33 \\ W. HELFRICH, Freie Universität Berlin, Institut für Theorie der Kondensierten \\ Materie, Arnimallee 3, D-1000 Berlin 33, West Germany
}

\begin{abstract}
Osmotic shrinkage of giant egg-lecithin vesicles was observed by phase-contrast microscopy. The vesicles remained or became spherical when shrinking. Small and thickwalled vesicles formed visible fingers attached to the sphere. The water permeability of the single bilayer was found to be $41 \mu \mathrm{m} / \mathrm{s}$. A variety of observations indicate that osmosis induces a parallel lipid flow between the monolayers of the bilayer, leading to a strong positive spontaneous curvature. They also suggest the formation of mostly submicroscopic daughter vesicles. The estimated coupling constant, $2 \cdot 10^{-6} \mathrm{~mol} / \mathrm{mol}$, is large enough to be biologically significant.
\end{abstract}

\section{INTRODUCTION}

Osmotic experiments on black lipid membranes of various materials, among them egg lecithin, were reviewed by Tien (1). The most recent measurements on egg lecithin are those of Fettiplace (2). Black films have the disadvantage of lateral tension (1) and residual organic solvents in the hydrocarbon region of the bilayer $(3,4)$, both of which may influence the permeability.

Studies of osmotically induced water permeation through vesicle walls have been rare. Reeves and Dowben (5) qualitatively described the behavior of thin-walled giant vesicles under osmotic shrinkage, noting that the spherical shape was maintained. They also measured the permeation of water and solute molecules via turbidity changes of vesicle suspensions (6). More recently, Blok et al. (7) studied the effect of cholesterol on the water permeation through liposomal lecithin walls determining the activation energy from the temperature dependence of the turbidity decay. Even if the number of lamellas composing the walls were known, evaluating permeabilities from turbidity changes does not seem unambiguous as the amount of scattered light depends both on the difference between the indices of refraction inside and outside a vesicle and on the distribution of the vesicles in size and shape.

In the following we report extensive studies of the osmotic shrinkage of giant lecithin vesicles which were individually observed under a phase contrast microscope. Most of the measurements were done on vesicles of minimum contour strength which were most likely unilamellar (8). A value for the permeability of the single bilayer to water is extracted from the data. This is possible because the vesicles remained or became spherical during osmotic shrinkage and their radii decreased linearly with time.

The thin-walled vesicles displayed recurrent destabilizations of the spherical shape when shrinking, but only if the radii were above a certain limit. In additional, qualitative studies we 
found small thick-walled vesicles to develop finger-like protuberances under osmotic shrinkage.

These and further observations are discussed in terms of a model which seems to explain them all consistently. It involves an intralamellar flow of lecithin molecules parallel to the flow of permeating water, a positive spontaneous curvature of the bilayer due to the resulting lipid imbalance, and the creation of visible protuberances or invisibly small daughter vesicles through the spontaneous curvature.

We think that our experiments are the first to show that water permeation through a lipid membrane induces lipid flow. From the limiting radius of destabilization and the assumption that the curvature of the daughters cannot be larger than that of sonicated vesicles we estimate the lipid-water coupling constant. A compatible lower limit of this quantity is more directly obtained from the visible deformations of thick-walled vesicles.

\section{EXPERIMENTAL}

Egg lecithin was purchased from Merck, D6100 Darmstadt, West Germany, and used without further purification. Large thin-walled vesicles with radii mostly between 5 and $50 \mu \mathrm{m}$ were prepared by simple swelling as described elsewhere $(9,10)$. Subsequently, they were allowed to stand for several days. As the flip-flop time of the common lecithins in the fluid phase is of the order of $10 \mathrm{~h}(1 \mathrm{l}-13)$ we could expect any lipid imbalance to vanish (14). To induce osmosis the external water was replaced by $\mathrm{NaCl}$ or glucose solutions of concentrations between 1.5 and $20 \mathrm{mM}$.

Two experimental procedures were used to study thin-walled vesicles. The first allowed observation during the onset of osmosis, thus enabling us to detect any sudden changes in shape and size or damage of the vesicle wall. The sample chamber consisted of a stainless steel cylinder $2 \mathrm{~mm}$ high, $15 \mathrm{~mm}$ in diameter, and covered with glass slides on top and bottom for microscopic observation. Two reservoirs were connected with the chamber, one containing distilled water and the other an aqueous solution of known concentration. The aqueous medium in the chamber could be replaced by raising or lowering a reservoir. It was verified by means of colored salt solutions that the exchange was practically complete. On the other hand, a few of the freely floating vesicles generally were not carried away during the exchange, but remained available for examination. As no particular phenomena were found to be associated with the onset of osmosis, a less tedious method was used for systematic observations. Lecithin vesicles were prepared by swelling in a test tube. After applying the osmotic gradient by adding salt or glucose solutions, a few microliters of the vesicle suspension were put between glass slides and observed under the microscope. Quantitative measurements of the shrinking process were then made on suitable vesicles either by direct observation of the radius of the vesicle through the ocular or by taking a sequence of microphotographs as shown in Figs. 1, 3, and 4. Thick-walled vesicles were prepared by shaking water and a little lecithin in a test tube. They were mainly spherical, the radii being only a few $\mu \mathrm{m}$. The osmotic experiments were done a few days afterwards. Only the second method of applying the osmotic gradient was used. All samples were prepared and all experiments performed at room temperature.

\section{RESULTS}

Most of our investigations were done with large thin-walled vesicles. Specimens for observation were selected by looking for vesicle contours of minimum contrast which, on the basis of earlier work (8), indicate that the wall consists of a single bilayer. Unilamellarity was confirmed by the nearly equal permeabilities to water calculated for most of these vesicles (see below).

The vesicles preserved their spherical shape during shrinkage, as already noted by Reeves and Dowben (5), or became spherical. Shrinkage was followed over ranges covering up to $60 \%$ 

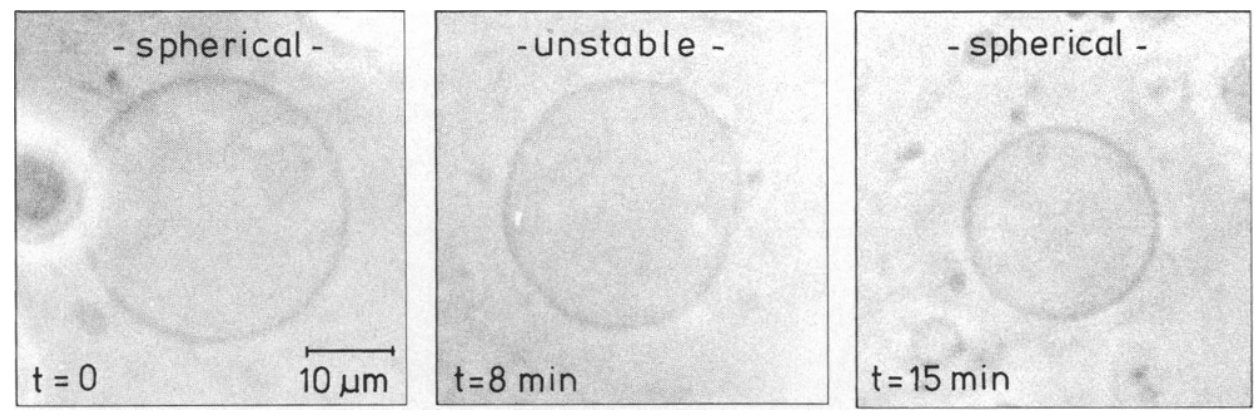

FIGURE 1 Osmotic shrinkage of a thin-walled spherical vesicle induced by a concentration difference of $\Delta c=15 \mathrm{mM}$. The shapes represent an initial spherical state $(t-0)$, an unstable state $(t=8 \mathrm{~min})$, and another spherical state $(t=15 \mathrm{~min})$.

of the initial radius. As a rule, we found a linear decrease of vesicle radius with time. Above a limiting radius of $\sim 10 \mu \mathrm{m}$ the vesicles exhibited periods of shape instability during osmotic shrinkage. The destabilization was gradual with increasing fluctuations around the spherical shape and culminated in marked deviations from the sphere that usually were not rotationally symmetric but irregular. The spherical shape was then reestablished. Fig. 1 shows a sequence of shapes of a vesicle, representing a spherical, an unstable, and again a spherical phase. A plot of vesicle radius vs. time together with a linear fit is shown in Fig. 2. The destabilizations were in general recurrent. The periods of shape instability were always shorter than the intervals of shrinkage in the spherical phase. Usually they did not noticeably disrupt the linear dependence of vesicle radius on time, as illustrated by Fig. 2 . The decrease in radius during a period of instability was always $<10 \%$.

Sometimes daughter vesicles much smaller than the mother, but well resolvable, were seen to be formed during an unstable phase. They seemed to remain attached to the mother. However, the nature of the connection, if any, could not be made out. Daughter vesicles were seen only around mothers whose initial radii were above the limiting value of $10 \mu \mathrm{m}$, which again suggests that destabilization was necessary for their formation.

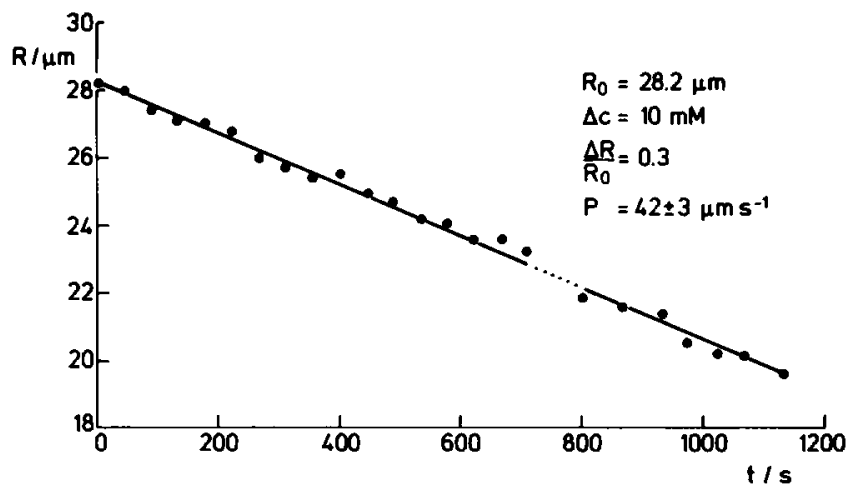

FIGURE 2 Radii of an osmotically shrinking spherical vesicle as a function of time. The drawn line represents a linear fit, the dotted portion indicating a period of shape instability. 

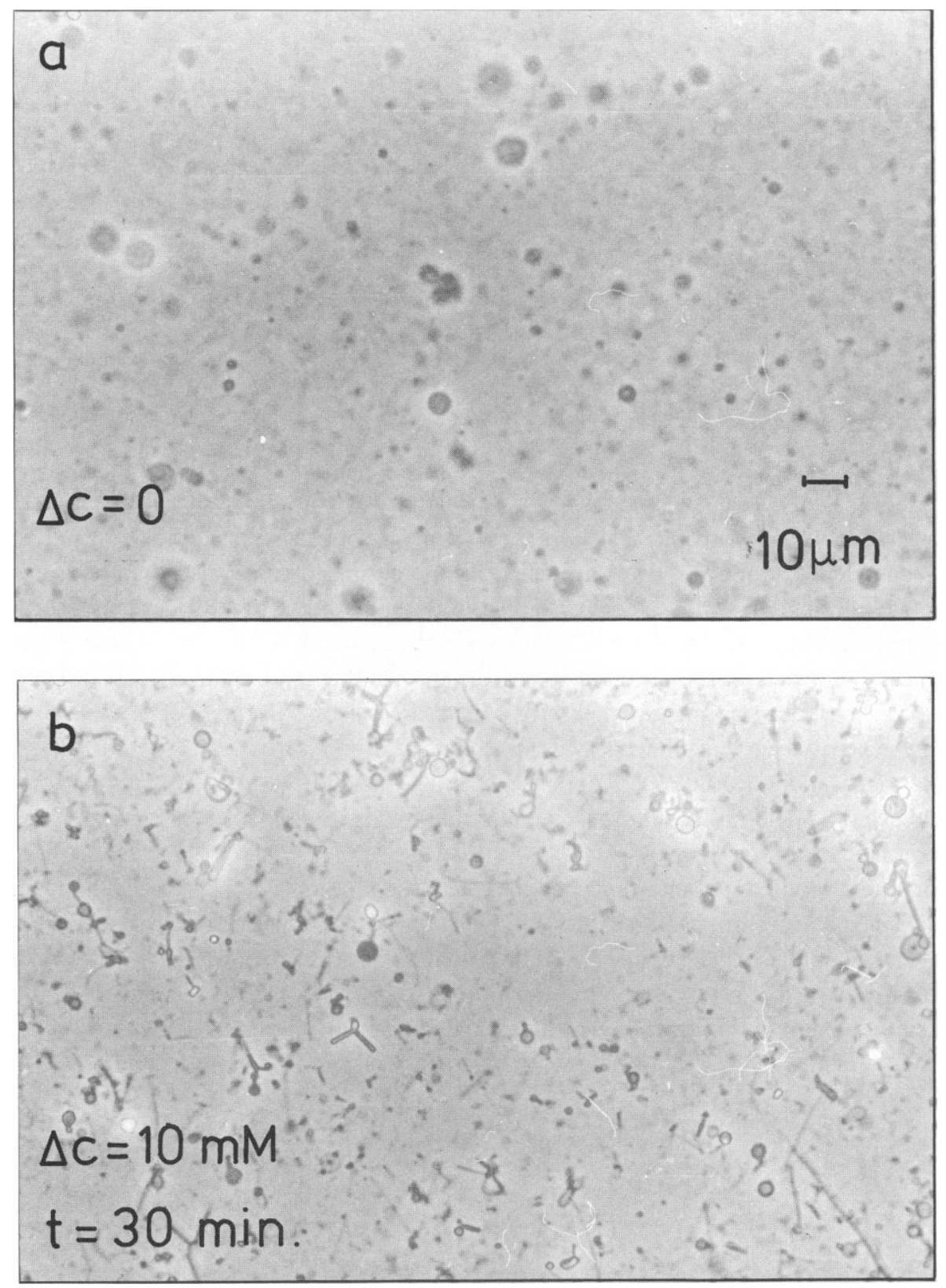

Figure 3 (a) Multilamellar vesicles as prepared in pure water and (b) after 30 min of osmotic shrinkage in $10 \mathrm{mM} \mathrm{NaCl}$ solution.

A few vesicles were observed which after an unstable phase displayed a smaller slope of radius vs. time than before. This indicates an increase of the solute concentration inside the vesicle, probably due to transitory pores which may be associated with shape destabilization. Occasionally, thin-walled vesicles exhibited no shrinkage at all or stopped it minutes after starting. The cessation of shrinking indicates the presence of long-lived or permanent holes. 
These may be pores in a single bilayer or passages in a bilamellar wall (15). Osmotically inactive vesicles were always spherical, which in conjunction with holes implies the absence of strong spontaneous curvature.

The typical effect of osmotic shrinkage on the small thick-walled vesicles is demonstrated in Fig. 3. Very small vesicles $\sim 1 \mu \mathrm{m}$ in diameter transformed into simple tubes. Because of the limit of resolution of the microscope it could not be completely ruled out that a few of them were unilamellar. Thick-walled vesicles with diameters up to $5 \mu \mathrm{m}$ were found to develop well discernible fingers which grew out of the shrinking sphere. As osmosis went on the fingers became thinner and longer at the same time. An example is shown in Fig. 4. In cases where more than one finger were created, their diameters appeared to be equal. A few vesicles whose walls were particularly thick had radii $>10 \mu \mathrm{m}$. They also displayed finger-like protuberances and, sometimes, temporary deviations from the spherical shape. The dimensions of the tubes seemed to increase with wall thickness. However, the osmotic shrinkage of thick-walled vesicles has not been investigated systematically.

\section{DISCUSSION}

\section{Derivation of the Permeability to Water}

The osmotic flow of water through a membrane is generally described by the law (1):

$$
j=-P \Delta c
$$

Here $j$ is the molar flux density and $\Delta c$ the difference of molar concentrations between the aqueous solutions. The material constant $P$ is called permeability and has the dimension of velocity. Eq. 1 applies if no osmotic pressure difference is built up between the solutions. Since in the present case osmosis acts on vesicles swollen in pure water, $\Delta c$ is identical to the concentration of the osmotic solutes $\left(\mathrm{Na}^{+}, \mathrm{Cl}^{-}\right.$, and glucose) in the medium outside the vesicle. For a spherical vesicle of radius $R$ we can write

$$
\frac{\mathrm{d} R}{\mathrm{~d} t}=-\alpha P \Delta c
$$

$\alpha$ being the molar volume of water, if we identify the instantaneous spherical surface area as seen under the micorscope with the osmotically active area. Eq. 2 describes a linear decrease of $R$ with time, in agreement with our observations.

The data of some shrunken vesicles and the permeability calculated by means of Eq. 2 are listed in Table I. Except the values of the last two rows, they are grouped around a mean value of $P=41 \mu \mathrm{m} / \mathrm{s}$. Since the thinnest-walled vesicles, as judged from the optical contrast of the contour, were selected for these experiments, we believe that this is the permeability of the single bilayer. The last two values, obtained from somewhat thicker-walled vesicles, are about half that of the single bilayer, so it seems likely that they belong to bilamellar walls.

The permeabilities measured on black films of egg lecithin quoted in the literature range from 17 to $100 \mu \mathrm{m} / \mathrm{s}$ at $36^{\circ} \mathrm{C}$ (1). Fettiplace (2) recently obtained $37 \mu \mathrm{m} / \mathrm{s}$ at $25^{\circ} \mathrm{C}$. He suggested that part of the large scatter of the permeability of egg lecithin to water is due to differences in chemical composition. In the light of this assumption the good agreement of our result with his may be fortuitous. 

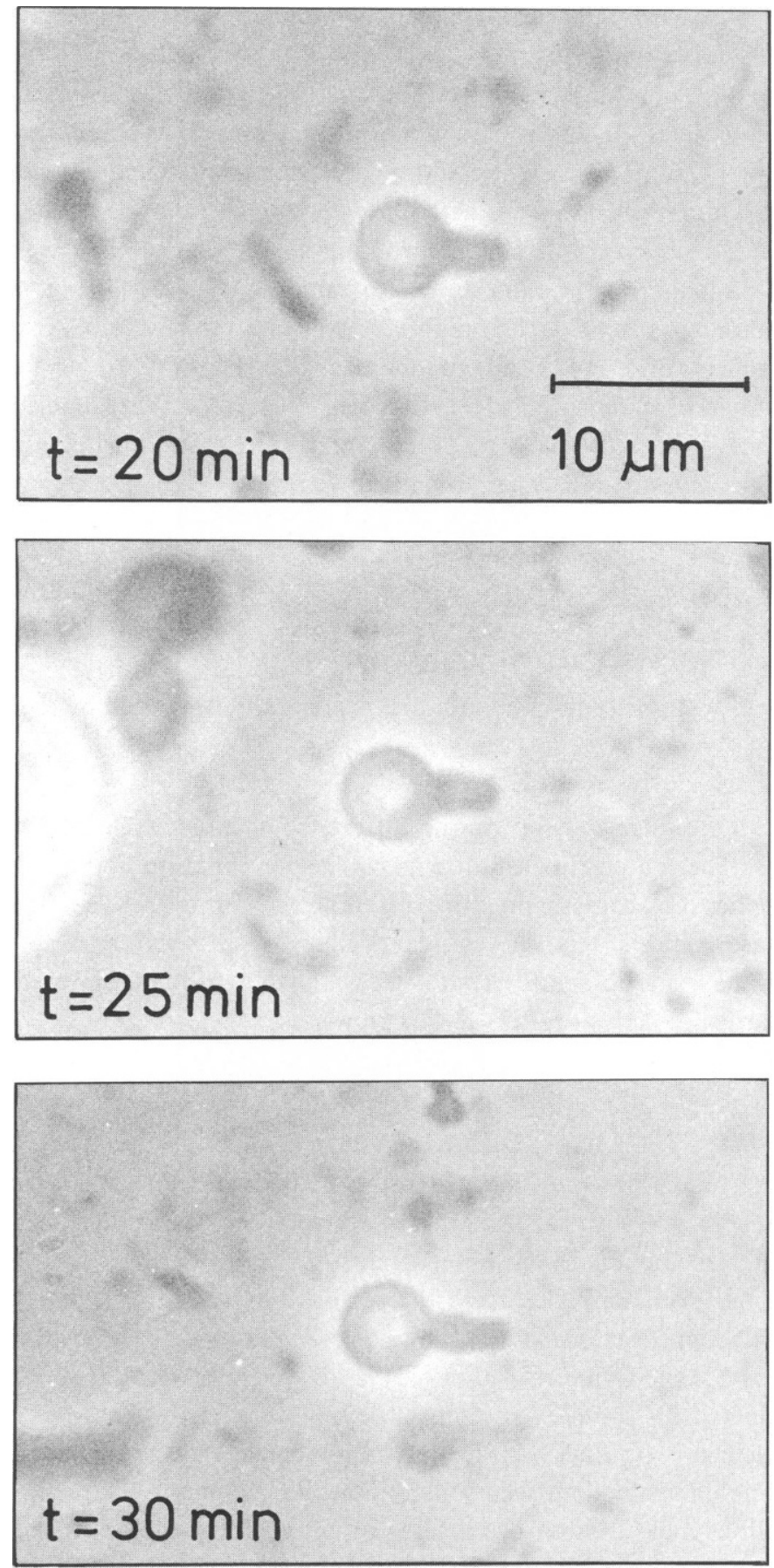

FIGURE 4 Tubular protuberance growing out of a multilamellar spherical vesicle under osmotic shrinking with a concentration difference of $\Delta c=10 \mathrm{mM}$. 

RADIUS $R(t)$ OF OSMOTICALLY SHRINKING THIN-WALLED VESICLES.

\begin{tabular}{ccll}
\hline$R_{0} / \mu \mathrm{m}$ & $\Delta \cdot 10^{3} / \frac{\mathrm{mol}}{\text { liter }}$ & $\Delta \mathrm{R} / \mathbf{R}_{\mathbf{0}}$ & $P / \frac{\mu \mathrm{m}}{s}$ \\
\hline 52 & 12.0 & 0.30 & $46 \pm 5$ \\
39 & 5.6 & 0.31 & $42 \pm 5$ \\
35 & 5.8 & 0.13 & $35 \pm 8$ \\
34 & 5.8 & 0.19 & $39 \pm 5$ \\
28 & 10.0 & 0.3 & $42 \pm 2$ \\
28 & 5.6 & 0.49 & $41 \pm 3$ \\
26 & 5.8 & 0.04 & $40 \pm 8$ \\
25 & 5.6 & 0.24 & $34 \pm 8$ \\
25 & 22.1 & 0.55 & $47 \pm 8$ \\
24 & 5.8 & 0.31 & $34 \pm 5$ \\
23 & 5.8 & 0.35 & $42 \pm 5$ \\
6.0 & 1.5 & 0.27 & $42 \pm 10$ \\
5.5 & 1.5 & 0.13 & $50 \pm 10$ \\
30 & 8.3 & 0.60 & $18 \pm 3$ \\
25 & -5.8 & 0.14 & $17 \pm 5$ \\
\hline
\end{tabular}

The first two columns give the initial values of vesicle radius $R_{0}$ and osmotic concentration difference $\Delta c$. The relative decrease of vesicle radius $\Delta R / R_{0}$ is related to the time of observation and does not necessarily indicate the radius at which shrinking stopped. All values of $P$ except the last two are assigned to unilamellar vesicles. The last two probably refer to vesicle walls consisting of two lamellas.

\section{Explanation of the Decrease of Vesicle Surface Area}

As osmotic shrinkage of thin-walled vesicles is described by Eq. 2 we must conclude that a loss of active wall area takes place during osmosis. It could be brought about by the dissolving of lipid molecules in the outer aqueous medium. However, the minute solubility of the common lecithins in water which is of the order $10^{-10} \mathrm{M}(16)$ can be shown to seriously hamper lipid transport of the required magnitude through water. While we do not preclude the possibility of lecithin molecules passing into solution and forming new, submicroscopic vesicles, we do not think this effect to be important.

A more likely mechanism that also explains the observation of protuberances is the direct formation of daughter vesicles from the wall of the mother. In the case of thin-walled vesicles, the daughters would normally have to be smaller than the resolving power of the microscope since visible new vesicles developed only during periods of instability. Any daughter vesicles must first be protuberances grown from, but still connected to, the mother. In the following we consider some geometric and elastic problems of the formation of protuberances and daughter vesicles under the action of spontaneous curvature. The origin of the spontaneous curvature is treated in the next subsection. The calculations apply to unilamellar walls.

A daughter vesicle must be separated from the mother at least by volume, though the two could still share the outer monolayer. This "semifission" may be expected to result in a narrow "neck" between mother and daughter and then to be followed by total separation, i.e., fission. The possibility of semifission will be mostly ignored. We do not know the shape of the postulated submicroscropic daughter vesicles. As to protuberances, two extreme forms can be imagined: the sphere and the cylinder. Spheres may also be lined up to form a string of pearls. 
The elastic theory of infinite cylinders, strings of pearls, and intermediate infinite shapes has been dealt with previously in a different context (17).

The water going into the protuberances and daughter vesicles should come from the mother. This requires a correction of Eq. 2 that depends on shape. If a single cylindrical protuberance of constant radius $r$ is formed (one is enough as its length is unlimited) we may write

$$
\frac{\mathrm{d} V_{\text {osmotic }}}{\mathrm{d} t}=\frac{\mathrm{d} V_{\text {mother }}}{\mathrm{d} t}+\frac{r}{2} 8 \pi R \frac{\mathrm{d} R}{\mathrm{~d} t}
$$

$r / 2$ being the ratio of volume to surface area of the cylinder. Expressing Eq. 2 in the form

$$
\frac{\mathrm{d} V_{\text {osmotic }}}{\mathrm{d} t}=-4 \pi R^{2} \alpha P \Delta C,
$$

we obtain

$$
\frac{\mathrm{d} R}{\mathrm{~d} t}\left(1-\frac{r}{R}\right)=-\alpha P \Delta C
$$

It is assumed in the derivation that the osmotically active area is identical to the area of the spherical mother vesicle. We could imagine a very narrow cylinder that breaks off from time to time to satisfy, at least approximately, this constraint imposed by the experimental results. In the case of continuous formation and separation of spherical daughter vesicles of constant radius $r$, the equivalent of Eq. 3 reads

$$
\frac{\mathrm{d} V_{\text {osmotic }}}{\mathrm{d} t}=\frac{\mathrm{d} V_{\text {mother }}}{\mathrm{d} t}+\frac{4 \pi}{3} r^{3} \frac{\mathrm{d} N}{\mathrm{~d} t}
$$

where $\mathrm{d} N=8 \pi R \mathrm{~d} R / 4 \pi r^{2}$ represents the differential of the number $N$ of daughters. Insertion of Eq. 4 now leads to

$$
\frac{\mathrm{d} R}{\mathrm{~d} t}\left(1-\frac{2}{3} \frac{r}{R}\right)=-\alpha P \Delta C
$$

For $r \ll R$, the corrected Eqs. 5 and 7 differ immeasurably from the original Eq. 2, which justifies the use of the latter in calculating the permeabilities. However, the small difference between Eqs. 5 and 7 has other consequences (see below).

Turning to elasticity, we start from the formula for the curvature-elastic energy per unit area, $g$, of fluid membranes (18). It may be written as

$$
g=1 / 2 \kappa\left(c_{1}+c_{2}-c_{0}\right)^{2}+\bar{\kappa} c_{1} c_{2}
$$

where $c_{1}$ and $c_{2}$ are the two principal curvatures, $c_{0}$ is the spontaneous curvature, and $\kappa$ and $\bar{\kappa}$ are elastic moduli, the dimension of which is energy. We assume the spontaneous curvature to be due solely to a lipid imbalance. It is then uniform throughout the membrane.

If water permeation produces little or no spontaneous curvature one would expect a sphere to become an ellipsoid after a small loss of volume (19). However, this was not observed in our experiments except during periods of shape instability. If on the other hand a small volume 
change is sufficient to produce a large positive spontaneous curvature $c_{0} \gg 2 / R$, the formation of cylindrical or spherical protuberances whose sum of curvatures $c_{1}+c_{2}$ roughly equals $c_{0}$ should be energetically favored. (Curvature like that of the sphere is counted positive.)

Goint into detail, we write down the total curvature-elastic energy $G$. Integration of Eq. 8 yields for a spherical mother vesicle of radius $R$ :

$$
G=2 \pi \kappa R^{2}\left(\frac{2}{R}-c_{0}\right)^{2}+4 \pi \bar{\kappa} .
$$

The second term is known to be independent of shape. If the mother vesicle shrinks and a cylindrical protuberance of radius $r$ is formed from the freed area, the time derivative of $G$ is given to a good approximation for $r \ll R$ by

$$
\frac{\mathrm{d} G}{\mathrm{~d} t}=4 \pi \kappa R^{2}\left(\frac{2}{R}-c_{0}\right) \frac{d(2 / R)}{\mathrm{d} t}+\frac{1}{2} \kappa \frac{\mathrm{d} A}{\mathrm{~d} t}\left[\left(\frac{2}{R}-c_{0}\right)^{2}-\left(\frac{1}{r}-c_{0}\right)^{2}\right],
$$

where $A=4 \pi R^{2}$ is the surface area of the mother. Eq. 10 is based on the assumption that the lateral compressibilty of the membrane is negligible. Its first term accounts for the change of curvature around the mother, the second for the transition of material into the cylinder. The derivation of $c_{0}$ does not enter because work is transferred only by changes of the actual curvatures $c_{1}$ and $c_{2}$. The radius of the cylindrical protuberance will be such as to maximize the release of elastic energy. This is the case for

$$
\frac{1}{r}=c_{0}
$$

The result may have been intuitively anticipated and justifies the omission of terms containing $\mathrm{d} r / \mathrm{d} t$ in Eq. 10. For $r \ll R$ there remains only one dominant term in Eq. 10, namely

$$
\frac{\mathrm{d} G}{\mathrm{~d} t} \approx \frac{1}{2} \kappa c_{0}^{2} \frac{\mathrm{d} A}{\mathrm{~d} t}
$$

According to the theory of elasticity of fluid membranes (18) the lateral tension due to curvature, which can be anisotropic, is given by

$$
\sigma_{11}=1 / 2 \kappa\left(c_{1}+c_{2}-c_{0}\right)\left(-c_{1}+c_{2}-c_{0}\right)+\lambda,
$$

for the force along the first principal axes acting on a section along the second. (Interchanging the subscripts 1 and 2 gives $\sigma_{22}$.) The term $\lambda$ represents a uniform and isotropic contribution. The tensions around the sphere and around the cylindrical protuberance are easily found to be

$$
\sigma_{R}=-\frac{1}{2} \kappa\left(\frac{2}{R}-c_{0}\right) c_{0}
$$

and

$$
\sigma_{r}=\lambda,
$$

both being isotropic. The pressures produced by these tensions must be equal as long as the 
enclosed volumes are connected, i.e.,

$$
2 \sigma_{R} / R=\sigma_{r} / r
$$

It follows that for $r \ll R$ and $r \approx 1 / c_{0}$

$$
\sigma_{\mathrm{R}} \approx 1 / 2 \kappa c_{0}^{2}, \quad \sigma_{r} \approx 0 .
$$

The lateral tension in the membrane around the mother may be substantial as will be discussed below.

If the continuous production of spherical protuberances instead of the growth of a single cylinder is considered, $1 / r$ has to be replaced by $2 / r$ in Eqs. $10-15$. No extra term is needed in the energy balance Eq. 10 for the supposedly narrow connection between mother and daughter. At least in theory this passage can be constructed such that $\left(c_{1}+c_{2}-c_{0}\right)$ vanishes everywhere in it (17). The change of elastic energy associated with the generation of one spherical protuberance, $\Delta G_{1}$, is readily found to be

$$
\Delta G_{1} \approx-8 \pi \kappa .
$$

At first sight it seems to make no difference for the decrease of elastic energy whether cylindrical or spherical protuberances are formed. However, assuming $1 / r=c_{0}$ for cylinders and $2 / r=c_{0}$ for spheres, we deduce from Eqs. 5 and 7 , that the volume going into protuberances of equal membrane area is slightly larger with spheres or strings of pearls than with cylinders. This volume could be entirely neglected in calculating $\mathrm{d} R / \mathrm{d} t$. Nevertheless, it causes an additional shrinkage of the mother, thus permitting a relatively very small additional growth to the protuberances. The difference of the correction factors of $\mathrm{d} A / \mathrm{d} t$ determines the apparent difference of the energy per unit area between spheres and cylinders, which is

$$
-\frac{r}{3 R} \frac{1}{2} \kappa c_{0}^{2}
$$

to first order in $r / R$. As an example we may think of transforming a string of pearls into a cylindrical protuberance. If $c_{1}+c_{2}$ of the protuberance and the total enclosed volume are kept constant in this process, part of the protruded area has to be forced back into the membrane around the mother.

The extra energy per pearl if a string of pearls is transformed into a cylinder is found from Eqs. 17 and 18 to be

$$
\frac{r}{3 R} 8 \pi \kappa
$$

Inserting $\kappa=2 \cdot 10^{-12} \mathrm{erg}$, as measured for the egg-lecithin bilayer (9), and assuming $R=10$ $\mu \mathrm{m}$ and $r=100 \AA$ (see below), one computes $2 \cdot 10^{-14} \mathrm{erg}$. The result seems interesting as it tells us that thermally accessible energies can probably open the constriction between two pearls or between a spherical protuberance and the mother.

It has been assumed from the beginning that the protuberances separate quickly from the mother, at least by volume, to form daughter vesicles. In the case of complete separation, i.e., fission, we have to add per daughter vesicle the "topological" energy $4 \pi \bar{\kappa}$. While this 
contribution becomes negligible for long enough cylinders, it may matter in the case of spheres. Considering the total curvature-elastic energy of spherical daughters, one obtains

$$
\frac{2}{r}=c_{0} /\left(1+\frac{\bar{\kappa}}{2 \kappa}\right)
$$

for the radius of maximum release of elastic energy. The modulus $\bar{\kappa}$ is unknown, but $0<\bar{\kappa}<\kappa$ may be expected to hold for egg lecithin (15). Therefore, the effect of $\bar{k}$ could be to favor the separation of strings of pearls rather than single spheres.

We have just presented a reason why spheres or strings of pearls should be preferred over cylinders in the generation of daughter vesicles. The associated constrictions can be a first step towards the separation of daughter vesicles. Nothing can be said about fission (or semifission) itself. It probably requires a certain activation energy. Moreover, dynamical aspects may have to be included in dealing with both the growth of protuberances and the separation of vesicles.

\section{Shape Destabilization and the Strength of Lipid-Water Flow Coupling}

In our model the spontaneous curvature is thought to be caused by a lipid imbalance between the monolayers. Adopting a linear approximation we may write

$$
c_{0}=\eta\left(\Delta \nu_{\text {tot }}-\Delta \nu_{\text {eq }}\right) / \nu_{0},
$$

where $\nu_{0}$ is the mean density of molecules per unit area and $\Delta \nu$ the difference between the densities in the outer monolayer and the inner one, all taken at the center plane between the monolayers. $\Delta \nu_{\text {oq }}$ is an equilibrium value proportional to the local sum of curvatures $\left(c_{1}+c_{2}\right)$ of the membrane. The total density difference $\Delta y_{\text {tot }}$ contains in addition any lipid imbalance. According to an earlier theory (20), the relevant proportionalities are taken to be

$$
\frac{(\Delta \nu)_{\text {oq }}}{\nu_{0}}=\frac{b}{2}\left(c_{1}+c_{2}\right)
$$

and

$$
\eta=\frac{6}{b}
$$

where $b$ is the thickness of the bilayer. We are interested in $\Delta \nu_{\text {tot }}$ as a function of curvatures. Insertion of Eqs. 22 and 23 in Eq. 21 yields

$$
\Delta \nu_{\mathrm{tot}}=\nu_{0} \frac{b}{2}\left(c_{1}+c_{2}\right)+\nu_{0} \frac{b}{6} c_{0}
$$

In protuberances with $c_{1}+c_{2} \approx c_{0}$, the first contribution is three times the second.

The lipid imbalance is assumed to be generated by a lipid flow parallel to the osmotic water flow. The coupling of lipid transport to water permeation may be expressed by

$$
\mathrm{d} n_{L} / \mathrm{d} t=K \mathrm{~d} n_{W} / \mathrm{d} t .
$$

Here $\mathrm{d} n_{w} / \mathrm{d} t$ is the flow of water molecules permeating through the membrane into the outer medium and $\mathrm{d} n_{L} / \mathrm{d} t$ the induced flow of lecithin molecules from the inner to the outer 
monolayer. For the permeation of water molecules we may write

$$
\frac{\mathrm{d} n_{W}}{\mathrm{~d} t}=-4 \pi R^{2} \frac{\mathrm{d} R}{\mathrm{~d} t} \frac{N_{A}}{\alpha},
$$

where $N_{A}$ is Avogadro's number.

It is possible to formulate a dynamic equilibrium of the increase of lipid imbalance by lipid-water coupling and its simultaneous decrease by the formation of protuberances and, eventually, daughter vesicles. This is particularly easy if we neglect the second term on the right hand side of Eq. 24. (Physically, this corresponds to $\eta=\infty$, i.e., lateral incompressibility of the monolayers.) Then the dynamic equilibrium takes the form:

$$
2 K \frac{\mathrm{d} n_{W}}{\mathrm{~d} t}+\frac{\mathrm{d} A}{\mathrm{~d} t} \nu_{0} \frac{b}{2}\left(c_{1}+c_{2}\right)=0,
$$

where we recall that $A$ is the area of the mother vesicle. The factor 2 in front of $K$ allows for the fact that the lipid transport lowers the lipid density of the inner monolayer as much as it raises that of the outer one.

Solving for the coupling constant, we obtain for $c_{1}+c_{2}=2 / r$, i.e., spherical protuberances,

$$
K=\frac{\nu_{0} b \alpha}{N_{A} r R} .
$$

The formula applies to cylinders if the right hand side is multiplied by $1 / 2$. Establishing a dynamic equilibrium is slightly more complex in the case of compressible lipids because $\Delta \nu_{\text {tot }}$ no longer equals $\Delta v_{\text {oq }}$. Assuming $r \sim 1 / R$ at all times, one calculates on the basis of Eq. 23 the additional correction factor $4 / 3$ for the right hand side of Eq. 28. It should be noted that in deriving Eq. 28, especially in using Eq. 26, we again assumed that the protuberances separate at least by volume from the mother once they are formed.

Eq. 28 shows the dynamic equilibrium to be characterized by a certain $r \cdot R$. As the radius $r$ can hardly be smaller than that of sonicated vesicles $(21,22)$, the radius $R$ should have an upper limiting value above which dynamic equilibrium cannot exist. It has just been argued that the daughter vesicles, attached or separate, should be spheres or strings of pearls. Cylinders seem to be preferred in the case of visible protuberances as demonstrated by the thick-walled vesicles but this may be a consequence of their small ratio of length to radius. Other complications might arise in the case of very large spontaneous curvatures near the limit just mentioned which should also be the limit of Hooke's law. If one assumes that each separate principal curvature, rather than the sum of principal curvatures, has an upper limiting value, spheres could accommodate two times more lipid imbalance than cylinders and would thus be favored even more.

We are now in a position to propose an explanation for the shape destabilization of vesicles which also supplies an estimate of the lipid-water coupling constant. The destabilization of large unilamellar vesicles, which occurred only above a certain size, may be caused by a growth of $c_{0}$, which can no longer be completely offset by the formation of daughter vesicles. A continuous buildup of spontaneous curvature will eventually lead to membrane rupture. Any damage of the bilayer permits a lipid exchange between the constitutive monolayers via its edges. This could lead to a more or less rapid breakdown of spontaneous curvature which 
would explain the transitory existence of nonspherical shapes, provided rupture is accompanied by an ejection of water (or a regrowth of active membrane area). The loss of water need not be so large as to be detectable. The pores should close after a while so that $c_{0}$ starts to grow again. Finally, the formation of invisible protuberances $(r \ll R)$ should be resumed and the spherical shape reestablished.

Accepting the model, one can estimate the lipid-water flow coupling constant from the observed $R_{\max }=10 \mu \mathrm{m}$ for shrinkage without periods of instability and the known $r_{\min }=100$ $\AA$ of sonicated vesicles (22). Inserting into Eq. 28 these values for $R$ and $r$, a bilayer thickness $b=40 \AA$, and a molecular area $1 / \nu_{0}=60 \AA^{2}$, one arrives at

$$
K \approx 2 \cdot 10^{-6} \text {. }
$$

It is also interesting to compute the lateral tension $\sigma_{R}$ in the membrane of the shrinking vesicle for $r=r_{\text {min }}$. From Eq. 16 and $\kappa=2 \cdot 10^{12} \mathrm{erg}(9)$ one obtains $\sigma_{R} \simeq 4 \mathrm{dyn} \mathrm{cm}^{-1}$ for spherical daughter vesicles. This seems a fairly high stress, which could indeed give rise to rupture.

Provided our model is correct and the estimated $K$ of the right order of magnitude, one cannot hope to see protuberances emerge from unilamellar vesicles. For $R=0.5 \mu \mathrm{m}$, the smallest resolvable size, one obtains from $R r=R_{\max } r_{\min }$ the still smaller radius $r=0.2 \mu \mathrm{m}$. In fact, no protuberances could be found with the smallest visible thin-walled vesicles despite an exhaustive search.

On the other hand, the small thick-walled vesicles developed resolvable protuberances under osmosis. The observation surprises in a way, as the number of lamellas should not affect shape changes except for their speed if the vesicle wall consists of independent bilayers. The apparent reduction of the lipid-water flow coupling constant could be due to a lipid exchange preventing the full buildup of lipid imbalance. The exchange might be mediated by resorbed daughter vesicles as well as by passages or necks connecting adjacent bilayers. It is also conceivable, though not probable, that the proximity of the bilayers in a wall permits a substantial flow of lecithin molecules through the intermediate water layers so that the full lipid imbalance develops only in the two outermost monolayers.

The effective coupling constants of thick-walled vesicles are estimated to be one to two powers of ten below the value deduced for the single bilayer. They can be estimated for initially spherical vesicles by inserting the radii of the shrunken mother and the cylindrical protuberance in Eq. 28. However, the method is rather crude as the conditions of vesicle separation and $r \ll R$ are not satisfied. In any event, the protuberances of thick-walled vesicles clearly confirm that water permeation produces positive spontaneous curvature.

We further note that the well-known flip-flop of lipid molecules which is a relaxation process may compete with the osmotically induced lipid flow if osmosis is performed very slowly. Since our experiments took no longer than several hours, whereas the flip-flop time is known to be of the order ten $10 \mathrm{~h}(11-13)$, we have neglected this backflow in our discussion. Moreover, the water permeability deduced from $R(t)$ did not noticeably depend on the speed of permeation in the range of concentrations used (see also below).

\section{CONCLUSION}

Our experiments show that osmotic shrinkage of giant egg-lecithin vesicles is a suitable method to measure the bilayer permeability to water. Its apparent independence of vesicle 
radius and osmotic gradient and the rather good agreement with black-film permeabilities (2) suggest that lateral tension, which we presume to be strong under osmotic shrinkage, has little or no influence on it.

An exciting result of our studies is the discovery that water permeation induces a considerable parallel flow of lecithin molecules between the two monolayers of a bilayer. In the case of large unilamellar vesicles, flow coupling was inferred indirectly, being part of a consistent model which can explain all observations. The finger-like protuberances developed by small thick-walled vesicles represent a more direct proof, but there the effective flow coupling constant comes out smaller by at least a power of ten. Interestingly, the transverse lipid flow in our experiments was faster than the flip-flop of lecithin molecules in the absence of osmosis. This is only possible if flip-flop is a process consisting of many steps and involving many collisions with water molecules.

We also deduced from our data that unilamellar giant vesicles form submicroscopic daughter vesicles when they shrink osmotically. Daughters of visible size were seen only in a few cases as a result of transitory shape destabilization. However, the fact that the osmotically active surface area diminshes together with the visible surface area during shrinkage cannot be explained but by a continuous loss of membrane material. It is not possible to decide whether the daughters are spherical or cylindrical or whether they fission completely from the mother or undergo semifission, i.e., retain a common outer monolayer. Even other mechanisms cannot be entirely excluded, such as the dissolving of lecithin molecules in water, as discussed above, or the growth of pieces of bilayer or cylindrical micelles from the outer monolayer. Different experimental methods, for instance electron microscopy, are needed to settle these questions.

Lipid-water flow coupling of the orders of magnitude estimated from our data could be biologically significant. Osmotically induced lipid imbalance may well be instrumental in pinocytosis, i.e., the fission of small daughter vesicles from a plasma membrane. It is surprising that this process common in the metabolism of cells (23) might be simulated by simple model membranes.

This work was supported by the Deutsche Forschungsgemeinschaft.

Received for publication $1 /$ June 1980 and in revised form 4 November 1980.

\section{REFERENCES}

1. TIEN, H. 1974. Bilayer Lipid Membranes. Marcel Dekker, Inc., New York.

2. FetTIPLACE, R. 1978. The influence of the lipid on the water permeability of artificial membranes. Biochim. Biophys. Acta. 513:1-10.

3. Henn, F. A., and T. E. Thompson. 1968. Properties of bilayer membranes separating two aqueous phases: composition studies. J. Mol. Biol. 31:227-235.

4. ANDREWS, D. M., and D. A. HAYDon. 1968. Electron microscope studies of lipid bilayer membranes. J. Mol. Biol. 32:149-150.

5. ReEves, J. P., and R. M. Dowben. 1969. Formation and properties of thin-walled phospholipid vesicles. J. Cell Physiol. 73:49-60.

6. ReEves, J. P., and R. M. Dowben. 1970. Water permeability of phospholipid vesicles. J. Membr. Biol. 3:123-141.

7. BoK, M. C., L. L. M. VAN DeENEN, and J. DE Gier. 1977. The effect of cholesterol incorporation of the temperature dependence of water permeation through liposomal membranes prepared from phosphatidylcholines. Biochim. Biophys. Acta. 464:509-518. 
8. Servuss, R. M., and E. Boroske. 1979. Lamellarity of artificial phospholipid-membranes determined by photometric phase-contrast microscopy. Phys. Lett. 69A:468-470.

9. Servuss, R. M., W. HARBICH, and W. Helfrich. 1976. Measurements of the curvature-elastic modulus of egg lecithin bilayers. Biochim. Biophys. Acta. 436:900-903.

10. Harbich, W., R. M. Servuss, and W. Helfrich. 1976. Optical studies of lecithin-membrane melting. Phys. Lett. 57A:294-296.

11. KornberG, R. D., and H. M. MC CONNELl. 1971. Inside-outside transitions of phospholipids in vesicle membranes. Biochemistry. 10:1111-1120.

12. De KruisfF, B., and E. J. J. VAN Zoelen. 1978. Effect of the phase transition of the transbilayer movement of dimyristoyl phosphatidylcholine in unilamellar vesicles. Biochim. Biophys. Acta. 51 1:105-115.

13. DE KRUIJfF, B., and K. W. A. WIRTZ. 1977. Induction of a relatively fast transbilayer movement of phosphatidylcholine in vesicles. Biochim. Biophys. Acta. 468:318-326.

14. Harbich, W., H. J. Deuling, and W. Helfrich. 1977. Optical observation of rotationally symmetric lecithin vesicle shapes. J. Physique. 38:727-729.

15. Harbich, W., R. M. Servuss, and W. Helfrich. 1978. Passages in lecithin-water systems. Z. Naturforsch. 33a:1013-1017.

16. TANFORD, C. 1973. The Hydrophobic Effect: Formation of Micelles and Biological Membranes. John Wiley \& Sons, London.

17. Deuling, H. J., and W. Helfrich. 1977. A theoretical explanation of the myelin shapes of red blood cells. Blood Cells. 3:713-720.

18. HeLFRICH, W. 1973. Elastic properties of lipid bilayers: theory and possible experiments. Z. Naturforsch 28c:693-703.

19. Deuling, H. J., and W Helfrich. 1976. The curvature elasticity of fluid membranes: a catalouge of vesicle shapes. J. Physique. 37:1335-1345.

20. Helfrich, W. 1974. Blocked lipid exchange in bilayers and its possible influence on the shape of vesicles. $Z$. Naturforsch. 29c:510-515.

21. Helfrich, W. 1974. The size of bilayer vesicles generated by sonication Phys. Lett. 50A:115-116.

22. Huang, C. 1969. Studies on phosphatidylcholine vesicles. Formation and physical characteriestics. Biochemistry. 8:344-352.

23. Bielka, H. 1973. Molekulare Biologie der Zelle. G. Fischer Verl, Stuttgart, West Germany. 